\title{
Insulin pump therapy, multiple daily injections, and cardiovascular mortality in 18168 people with type 1 diabetes: observational study
}

\author{
Isabelle Steineck, Jan Cederholm, ${ }^{1}$ Björn Eliasson, ${ }^{3}$ Araz Rawshani, ${ }^{4}$ Katarina Eeg-Olofsson, ${ }^{3}$ \\ Ann-Marie Svensson, ${ }^{4}$ Björn Zethelius, ${ }^{5,6}$ Tarik Avdic, ${ }^{4}$ Mona Landin-Olsson, ${ }^{7}$ Johan Jendle, ${ }^{8}$ Soffia \\ Gudbjörnsdóttir ${ }^{3,4}$ the Swedish National Diabetes Register
}

${ }^{1}$ Department of Endocrinology,

Aarhus University Hospital,

Aarhus Denmark

${ }^{2}$ Department of Public Health and Caring Sciences/Family and Preventive Medicine, Uppsala University, Uppsala, Sweden 3Institute of Medicine,

Sahlgrenska University Hospital, University of Gothenburg,

Gothenburg, Sweden

${ }^{4}$ National Diabetes Register,

Centre of Registers,

Gothenburg, Sweden

${ }^{5}$ Department of Public Health and Caring Sciences/Geriatrics, Sweden

${ }^{6}$ Medical Products Agency, Uppsala, Sweden

${ }^{7}$ Department of Clinical Science, Lund University, Lund, Sweden

${ }^{8}$ Faculty of Health Sciences and Medicine, Örebro University,

Örebro, Sweden

Correspondence to: I Steineck isabelle.steineck@iname.com

Additional material is published online only. To view please visit the journal online (http://dx.doi. org/10.1136/bmj.h3234)

Cite this as: $B M J$ 2015;350:h3234 doi: 10.1136/bmj.h3234

Accepted: 11 June 2015
Uppsala University, Uppsala,

\section{ABSTRACT}

\section{OBJECTIVE}

To investigate the long term effects of continuous subcutaneous insulin infusion (insulin pump therapy) on cardiovascular diseases and mortality in people with type 1 diabetes.

DESIGN

Observational study.

SETTING

Swedish National Diabetes Register, Sweden 2005-12. PARTICIPANTS

18168 people with type 1 diabetes, 2441 using insulin pump therapy and 15727 using multiple daily insulin injections.

\section{MAIN OUTCOME MEASURES}

Cox regression analysis was used to estimate hazard ratios for the outcomes, with stratification of propensity scores including clinical characteristics, risk factors for cardiovascular disease, treatments, and previous diseases.

RESULTS

Follow-up was for a mean of 6.8 years until December 2012, with 114135 person years. With multiple daily injections as reference, the adjusted hazard ratios for insulin pump treatment were significantly lower: 0.55 ( $95 \%$ confidence interval 0.36 to 0.83 ) for fatal coronary heart disease, 0.58 (0.40 to 0.85 ) for fatal cardiovascular disease (coronary heart disease or stroke), and 0.73 (0.58 to 0.92) for all cause mortality. Hazard ratios were lower, but not significantly so, for fatal or non-fatal coronary heart disease and fatal or non-fatal cardiovascular disease. Unadjusted absolute differences were 3.0 events of fatal coronary heart

\section{WHAT IS ALREADY KNOWN ON THIS TOPIC}

In patients with diabetes, both hyperglycaemia and hypoglycaemia are risk factors for cardiovascular disease (coronary heart disease or stroke)

Continuous subcutaneous infusion of insulin with a pump could result in fewer episodes of hyperglycaemia and hypoglycaemia than multiple daily injections and provide better glycaemic control

\section{WHAT THIS STUDY ADDS}

Treatment of type 1 diabetes with an insulin pump is associated with significantly lower adjusted hazard ratios for fatal coronary heart disease, fatal cardiovascular disease, and all cause mortality, as well as non-significant reduction in hazard ratios for non-fatal or fatal cardiovascular disease

Patient education and frequency of blood glucose monitoring might have influenced the observed association disease per 1000 person years; corresponding figures were 3.3 for fatal cardiovascular disease and 5.7 for all cause mortality. When lower body mass index and previous cardiovascular diseases were excluded, results of subgroup analyses were similar to the results from complete data. A sensitivity analysis of unmeasured confounders in all individuals showed that an unmeasured confounders with hazard ratio of 1.3 would have to be present in $>80 \%$ of the individuals treated with multiple daily injections versus not presence in those treated with pump therapy to invalidate the significantly lower hazard ratios for fatal cardiovascular disease. Data on patient education and frequency of blood glucose monitoring were missing, which might have influenced the observed association.

\section{CONCLUSION}

Among people with type 1 diabetes use of insulin pump therapy is associated with lower cardiovascular mortality than treatment with multiple daily insulin injections.

\section{Introduction}

Nobody disputes the fact that type 1 diabetes increases the risk of death from cardiovascular diseases. A recent study from the Swedish National Diabetes Register showed that individuals with type 1 diabetes who have a glycated haemoglobin $\mathrm{A} 1 \mathrm{c}\left(\mathrm{HbA}_{1 \mathrm{c}}\right)$ concentration of $6.9 \%$ ( $52 \mathrm{mmol} / \mathrm{mol}$ or lower have a risk of death from any cause and from cardiovascular causes twice as high as the risk in the general population; the risks are several times higher among patients with higher $\mathrm{HbA}_{1 \mathrm{c}}$ concentrations. ${ }^{1}$ The Diabetes Control and Complication Trial and other recent studies have shown that tight glucose control reduces the risk, delays the onset, and slows the progression of complications. ${ }^{23}$ Other studies have shown that both hyperglycaemia and hypoglycaemia are risk factors for cardiovascular disease. ${ }^{4}$

Continuous subcutaneous insulin infusion involves connection of a catheter on the outside of the body to an insulin pump that is programmed to supply the body's basal needs. The person with the pump administers doses to cover meals and correct blood glucose concentrations. Many pumps these days have a bolus wizard that calculates how much insulin the person needs, taking expected carbohydrate intake, current blood glucose concentrations and previously still active insulin into consideration. Pumps can provide an accurate history of insulin use through their menus. Often 
this history can be uploaded and displayed as a graph for purposes of trend analysis.

Insulin pumps can result in fewer episodes of hyperglycaemia and hypoglycaemia than multiple daily injections. ${ }^{56}$ Some studies have shown that insulin pumps provide better glycaemic control than multiple daily injections. ${ }^{7}$ Three meta-analyses of randomised controlled trials investigated the association between treatment with a pump and the occurrence of hyperglycaemia. All three analyses found that insulin pump treatment was associated with improvement in $\mathrm{HbA}_{1 \mathrm{c}}$ compared with multiple daily injections, without a higher rate of hypoglycaemia. ${ }^{8-10}$ Fredheim and colleagues found that insulin pump therapy reduced the rate of severe hypoglycaemia by $27 \%$ compared with multiple daily injections. ${ }^{11}$ Given the importance of glycaemic control, ${ }^{1-3}$ and the presumed advantages of insulin pump treatment, it is important to investigate whether use of insulin pumps affects the risk of cardiovascular mortality.

In 2013 in Swedish people with type 1 diabetes, one out of every four women and one out of every five men used insulin pump treatment. Over half of all Swedish children with type 1 diabetes also use insulin pumps. ${ }^{7}$ Sceptics argue that subcutaneous infusion of insulin by a pump could increase costs of treatment and cause practical problems for people with diabetes. Because of the scarcity of data, the relative risk for cardiovascular disease of associated with the treatments is unknown.

Sweden offers excellent opportunities for studying individuals with type 1 diabetes. All affected people are treated at negligible personal cost. About $95 \%$ of all individuals with type 1 diabetes have been entered in the National Diabetes Register, which includes detailed clinical data from each appointment. The register can be linked via Sweden's unique personal identity number to the cause of death, inpatient, socioeconomic, and other population based registers. Our primary aim was to analyse the effect of insulin pump treatment on cardiovascular mortality.

\section{Methods}

\section{Swedish National Diabetes Register}

The Swedish National Diabetes Register was initiated in 1996 as a caregiver tool for local quality assurance and to provide feedback as part of diabetes care. Trained doctors and nurses report annually to the register, ${ }^{12}$ either online or through clinical record systems; no stringent criteria exist for how often patients visit an outpatient clinic. Information is collected during appointments at hospital outpatient clinics and primary healthcare centres nationwide. Several previous reports have been published concerning trends in risk factor control and risk prediction based on the register, ${ }^{1314}$ including a more detailed description of the register and the Swedish healthcare system for patients with diabetes. ${ }^{15}$

\section{Patient involvement}

There was no patient involvement in this study. The work within the Swedish National Diabetes Register, as this article, is done in a continuous but informal dialogue with people with diabetes.

\section{Participants}

We included 18168 people with type 1 diabetes entered in the Swedish National Diabetes Register for whom data were available about the use of insulin pump therapy or multiple daily injections. A total of 2441 people were being treated with insulin pump therapy during the study period from baseline to the final year, and 15727 were treated with multiple daily injections during the whole study period to final year. Type 1 diabetes was epidemiologically defined as all patients who received insulin treatment only (for diabetes mellitus) and were aged under 30 at onset, almost all of whom had been reported by outpatient clinics from about 90 Swedish hospitals. Baseline appointments took place in 2005-07 with follow-up until 31 December 2012. Treatment with insulin pump has been documented in the register since the year 2004. All individuals were recruited from the Swedish National Diabetes Register with no exclusion criteria.

\section{Examinations at baseline and the end of the study} Clinical characteristics at baseline included type of glucose lowering treatment, age, duration of diabetes, sex, HbA1c, systolic blood pressure, diastolic blood pressure, height, weight, waist circumference, physical activity, smoking habits, total cholesterol, high density lipoprotein cholesterol, triglycerides, microalbuminuria, plasma creatinine, use of antihypertensive drugs, lipid lowering drugs and acetylsalicylic acid (aspirin), atrial fibrillation, and histories of cardiovascular disease, heart failure and atrial fibrillation, Furthermore, baseline yearly income (in Swedish kroner), marital status (single, married, divorced, or widowed) and educational level (lower (up to school year 9), intermediate (years 10-12 of upper secondary school), and higher (college/university)) were obtained from the Longitudinal Integration Database for Health Insurance and Labour Market Studies, Statistics Sweden. Body mass index (BMI) was calculated as weight/height2. Waist circumference $(\mathrm{cm})$ was measured at the height of the navel. Physical activity was graded as low (no activity or less than once a week) or higher (twice or more a week). Smoking was defined as one or more cigarettes a day, one pipe a day, or having quit within the past three months. The Swedish standard for recording blood pressure as used by the Swedish National Diabetes Register is the average ( $\mathrm{mm} \mathrm{Hg}$ ) of two supine readings (Korotkoff sounds I-V) with a cuff of appropriate size after at least 5 minutes of rest. Analyses of HbA1c were quality assured nationwide by regular calibration with the HPLC Mono-S method and then converted to mmol/ mol. ${ }^{16}$ Albuminuria was classed as urine albumin excretion $>20 \mu \mathrm{g} / \mathrm{min}$ on two out of three consecutive tests (microalbuminuria or macroalbuminuria). A history of cardiovascular disease was defined the same way as for the outcome; ICD-10 (international classification of diseases, 10th revision) code I50 for heart failure; code I48 for atrial fibrillation; C00-C097 for all cancer; codes 
K70-74 for liver disease; and codes F20-29 and F30-39 for mental disorders.

We estimated updated mean $\mathrm{HbA}_{1 \mathrm{c}}$ during the study period using all values from baseline until the year before an event occurred during the study or otherwise from baseline until 31 December 2012. Change in $\mathrm{HbA}_{1 \mathrm{c}}$ during the study period was estimated as the difference between baseline and final measurements, the latter estimated as the value before the year of an event or otherwise the value in 2012. Hypoglycaemic attacks that required a hospital admission, with ICD-10 codes for hypoglycaemia and coma from the hospital discharge register, were entered during the study period from baseline until 31 December 2012.

\section{Follow-up and definition of endpoints}

All individuals were monitored from the baseline examination until death or the first incident or until 31 December 2012. The mean follow-up period was 6.8 years, with a total of 114135 person years. The major primary endpoints were fatal or non-fatal coronary heart disease, fatal or non-fatal cardiovascular disease, fatal cardiovascular disease, and total mortality. Non-fatal coronary heart disease was defined as non-fatal myocardial infarction (ICD-10 code I21), unstable angina (ICD-10 code I20.0), percutaneous coronary intervention, and/or coronary artery bypass grafting. Fatal coronary heart disease was defined as ICD-10 codes I20-I25. Stroke was defined as fatal or non-fatal cerebral infarction, intracerebral haemorrhage, or unspecified stroke (ICD-10 codes I61, I63, I64). Cardiovascular disease was defined as the composite of coronary heart disease or stroke, whichever came first. A secondary endpoint was mortality from non-cardiovascular disease.

A history of heart failure was defined as ICD-10 code I50, and atrial fibrillation before the study start was defined as ICD-10 code I48. All events were obtained by linking to the Swedish cause of death and hospital discharge registers, a reliable validated alternative to revised hospital discharge and death certificates. ${ }^{17} 18$

\section{Statistical analysis}

We applied five imputations using the Markov chain Monte Carlo technique for missing data in the sample of 18168 individuals, using the SAS MI and MIANALYSE procedures. ${ }^{19}$ We recorded baseline clinical features as mean values (SD) or frequencies (\%) of each multiple imputed variable in the two treatment groups (insulin pump therapy or multiple daily injections) and calculated significance for crude differences between the two groups with Student's $t$ test or $\chi^{2}$ test. We used crude Kaplan-Meier curves for all outcomes to compare the two groups with log rank test and for observed hypoglycaemic episodes during study follow-up.

We estimated a propensity score for treatment with pump with logistic regression as the conditional probability of being treated with pump given the baseline characteristics, ${ }^{20} 21$ including the covariates age, sex, duration of diabetes, history of cardiovascular disease, heart failure, atrial fibrillation, baseline $\mathrm{HbA}_{1 \mathrm{c}}$, systolic and diastolic blood pressure, BMI, total and high density lipoprotein cholesterol, triglycerides, cumulative microalbuminuria, creatinine, renal insufficiency, smoking, physical activity, antihypertensive drugs, lipid lowering drugs and aspirin, educational levels, yearly income, marital status and baseline years. We calculated $\mathrm{P}$ values for differences between the two treatment groups after adjustment with the propensity score, including all 36 variables, estimated by generalized linear models (link id for continuous data and link logit for dichotomous data). We also computed standardised differences between the two groups; a difference of less than $10 \%$ was considered to be satisfactory. ${ }^{21} 22$ The distribution of the propensity score stratified in fifths was calculated for the two treatment groups, as well as the number of outcomes by each fifth of the score (appendix table A).

We used Cox regression analysis to estimate hazard ratios with $95 \%$ confidence intervals for outcomes comparing insulin pump treatment with multiple daily injections. Covariate adjustment was performed by stratification with fifths of the propensity score. ${ }^{20} 21$

The proportional hazard assumption of the Cox regression analyses was tested by adding an interaction term of the predictor and log time and by analysing Schoenfeld residuals-both were found to be nonsignificant and satisfied the proportional hazard assumption. ${ }^{23}$ Interactions between the two treatment groups and all covariates included in the propensity score were analysed by means of maximum likelihood estimation; no interactions were found between any covariates.

Unmeasured confounders can affect the results if they are unrelated to, or not fully accounted for by, measured confounders or if they affect the decision to use insulin pump treatment and not multiple daily injections (treatment allocation bias). We therefore performed a sensitivity analysis by quantifying the effects of a hypothetical unmeasured confounder when comparing individuals treated with insulin pump therapy and multiple daily injections with an algorithm as presented by Lin and colleagues, ${ }^{24}$ yielding progressive changes of the hazard ratios observed for the analysed outcome with presence of an unmeasured covariate of 1.3 or 1.4 and present more frequently in one of the groups (injection) than the other. ${ }^{25-28}$

All statistical analyses were performed with SAS version 9.3 (SAS Institute, Cary, NC). A two sided $\mathrm{P}<0.05$ was considered significant.

\section{Results}

Table 1 shows baseline crude characteristics of the two groups (insulin pump treatment or multiple daily injections). The group treated with insulin pump therapy was somewhat younger with similar durations of diabetes, slightly lower systolic blood pressure, fewer men, fewer smokers, greater physical activity, less albuminuria, less renal insufficiency, less use of antihypertensive drugs, lipid lowering drugs, and aspirin, less history of cardiovascular disease and heart failure, better educated, and more likely to be married. Standardised differences between the two groups were sufficiently low and clearly below $10 \%$ for all 


\begin{tabular}{|c|c|c|c|c|c|}
\hline & Pump $(n=2441)$ & MDIs $(n=15727)$ & $\begin{array}{l}\text { Standardised } \\
\text { difference (\%)‡ }\end{array}$ & Pvalue* & Pvaluet \\
\hline Age (years) & $38(13)$ & $41(15)$ & 3.4 & $<0.001$ & 0.18 \\
\hline Onset age (years) & $16(8)$ & $13(7)$ & 1.8 & $<0.001$ & 0.7 \\
\hline Duration (years) & $25(12)$ & $26(15)$ & 2.3 & $<0.001$ & 0.3 \\
\hline$\%$ of men & 45.0 & 57.1 & 1.2 & $<0.001$ & 0.6 \\
\hline $\mathrm{HbA}_{\mathrm{cc}}(\mathrm{mmol} / \mathrm{mol})$ & $63(13)$ & $64(14)$ & 0.4 & 0.3 & 0.8 \\
\hline $\mathrm{HbA}_{1 \mathrm{c}}(\%)$ & $7.9(1.3)$ & $8.0(1.3)$ & 0.4 & 0.3 & 0.8 \\
\hline Systolic blood pressure $(\mathrm{mm} \mathrm{Hg})$ & $126(15)$ & $128(16)$ & 1.7 & $<0.001$ & 0.5 \\
\hline Diastolic blood pressure $(\mathrm{mm} \mathrm{Hg})$ & $73(8)$ & $73(9)$ & 0.7 & 0.2 & 0.7 \\
\hline \% taking antihypertensives & 32.0 & 36.7 & 1.1 & $<0.001$ & 0.7 \\
\hline Total cholesterol (mmol/L) & $4.7(0.9)$ & $4.8(0.9)$ & 2.4 & $<0.001$ & 0.3 \\
\hline HDL cholesterol (mmol/L) & $1.6(0.5)$ & $1.6(0.5)$ & 2.8 & 0.2 & 0.3 \\
\hline Triglycerides (mmol/L) & $1.0(0.7)$ & $1.1(0.8)$ & 2.7 & $<0.001$ & 0.6 \\
\hline$\%$ taking lipid drugs & 21.0 & 26.4 & 0.6 & $<0.001$ & 0.8 \\
\hline $\mathrm{BMI}\left(\mathrm{kg} / \mathrm{m}^{2}\right)$ & $25.3(3.8)$ & $25.4(4.1)$ & 0.2 & 0.061 & 0.8 \\
\hline$\%$ with low physical activity & 21.8 & 24.0 & 0.4 & 0.01 & 0.7 \\
\hline$\%$ of smokers & 10.5 & 13.5 & 2.5 & $<0.001$ & 0.4 \\
\hline \% taking aspirin & 15.0 & 18.8 & 1.4 & $<0.001$ & 0.6 \\
\hline Creatinine $(\mu \mathrm{mol} / \mathrm{L}) \S$ & $82(46)$ & $85(55)$ & 0.3 & 0.009 & 0.5 \\
\hline$\%$ with eGFR $<60$ & 10.4 & 11.7 & 0.2 & 0.04 & 0.7 \\
\hline$\%$ with cumulative albuminuria & 20.7 & 24.0 & 2.5 & $<0.001$ & 0.3 \\
\hline$\%$ with previous CVD & 5.4 & 8.0 & 1.8 & $<0.001$ & 0.5 \\
\hline$\%$ with previous heart failure & 0.9 & 2.3 & 3.2 & $<0.001$ & 0.9 \\
\hline$\%$ with previous atrial fibrillation & 0.6 & 1.0 & 0.7 & 0.05 & 0.9 \\
\hline$\%$ with previous cancer & 0.9 & 1.2 & 1.2 & 0.2 & 0.4 \\
\hline$\%$ with previous liver diseases & 0.3 & 0.2 & 0.3 & 0.5 & 0.7 \\
\hline$\%$ with previous mental disorders & 2.0 & 1.8 & 2.6 & 0.6 & 0.3 \\
\hline$\%$ with low education level & 9.8 & 19.0 & 3.0 & $<0.001$ & 0.6 \\
\hline$\%$ with medium education level & 52.9 & 53.5 & 1.9 & 0.6 & 0.4 \\
\hline$\%$ with high education level & 37.3 & 27.6 & 3.1 & $<0.001$ & 0.3 \\
\hline Annual income $\times 10^{-2}(\mathrm{SK})$ & $1697(1184)$ & $1702(1203)$ & 3.9 & 0.9 & 0.10 \\
\hline$\%$ single & 51.6 & 51.5 & 1.8 & 0.9 & 0.5 \\
\hline$\%$ married & 40.3 & 36.5 & 2.6 & $<0.001$ & 0.3 \\
\hline$\%$ divorced & 7.4 & 10.3 & 0.3 & $<0.001$ & 0.9 \\
\hline$\%$ widow & 0.7 & 1.7 & 1.9 & $<0.001$ & 0.7 \\
\hline$\%$ with baseline year 2005 & 69.4 & 66.9 & 1.7 & 0.01 & 0.5 \\
\hline$\%$ with baseline year 2006 & 21.7 & 21.2 & 2.0 & 0.6 & 0.4 \\
\hline$\%$ with baseline year 2007 & 11.4 & 9.3 & 0.4 & 0.001 & 0.7 \\
\hline
\end{tabular}

covariates. The distribution of the propensity score among the fifths (see appendix table A) shows a satisfactory overlap between the two treatment groups, as well as a sufficient number of outcomes by each fifth of the score. The mean percentage of missing data among all 36 variables was 5\%-the highest proportions were for physical activity (32\%), total cholesterol (25\%), high density lipoprotein cholesterol (22\%), triglycerides (21\%), creatinine (11\%), microalbuminuria $(10 \%)$, and BMI (9\%).

All individuals were monitored for a total of 114135 person years over a mean follow-up period of 6.8 years. There were 1423 cases of fatal or non-fatal cardiovascular disease during the study period. The incidence rate for cardiovascular disease was 1.1 cases a year or 12.5 cases per 1000 person years.
Figure 1 shows Kaplan-Meier crude survival curves for all outcomes during follow-up, with significant differences at log rank test. Table 2 shows adjusted hazard ratios for the outcomes with insulin pump treatment, with multiple daily injections as the reference group. After adjustment for the propensity score, including all variables presented in table 1, insulin pump treatment was associated with a hazard ratio of 0.81 ( 0.66 to 1.01 ) for fatal or non-fatal coronary heart disease, 0.55 (0.36 to 0.83 ) for fatal coronary heart disease, 0.58 ( 0.40 to 0.85 ) for fatal cardiovascular disease, and 0.73 (0.58 to 0.92) for all cause mortality. Non-significantly lower hazard ratios were found for fatal or non-fatal cardiovascular disease. Differences in absolute rates (per 1000 person years) were 4.5 for fatal/non-fatal coronary heart disease, 4.8 for fatal/non-fatal cardiovascular 

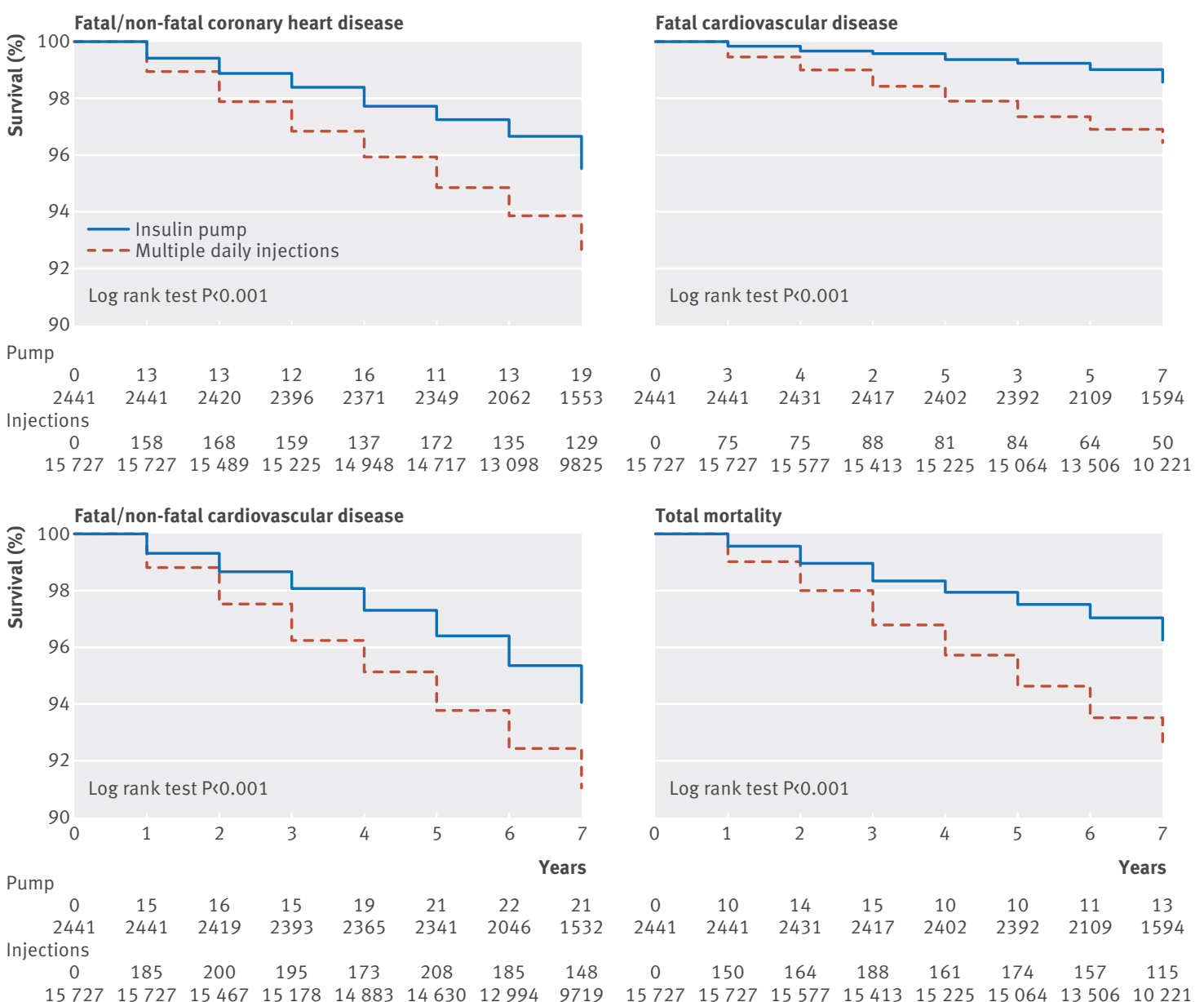

Fig 1 | Kaplan-Meier crude survival curves in 18168 individuals with type 1 diabetes according to treatment with insulin pump therapy or multiple daily injections. No of cases and individuals at risk are given in each group

disease, 3.3 for fatal cardiovascular disease, and 5.7 for total mortality.

We performed a sensitivity analysis with a hypothetical unmeasured confounder. Table 3 shows, for example, how a binary confounder with a hazard ratio of 1.3 or 1.4 would change the hazard ratio of 0.58 (95\% confidence interval 0.40 to 0.85 ) for fatal cardiovascular disease, depending on its prevalence in the group. If no patient treated with insulin pump therapy is exposed to the confounder (prevalence of 0.0 ), with a hazard ratio of 1.3, the difference between groups would not be significant (0.72, 0.50 to 1.05$)$, assuming that $80 \%$ were exposed in the insulin pump group. The corresponding figure for a confounder with a hazard ratio of 1.4 would be $60 \%(0.72,0.50$ to 1.05$)$.

\section{Complementary analyses}

We carried out a subgroup analysis consisting of 16427 individuals with $\mathrm{BMI} \geq 18$ and no history of cardiovascular disease, heart failure, or atrial fibrillation (see appendix table B).

The same pattern of results was seen as in all individuals, with borderline significant hazard ratios of $0.77(\mathrm{P}=0.046)$ for fatal or non-fatal coronary heart disease. Hazard ratios were significant for fatal coronary heart disease $0.39(\mathrm{P}=0.007)$, fatal cardiovascular disease $(0.48, \mathrm{P}=0.01)$, and total mortality $(0.75$, $\mathrm{P}=0.04)$. The hazard ratios for the other endpoints were not significant.

Another subgroup analysis of the 10282 patients with complete data for all variables in table 1 also showed similar results to the results for all patients (tables $\mathrm{C}$ and $\mathrm{D}$ in appendix). Differences between the pump therapy and injection groups for all variables after adjustment with a propensity score were not significant-standardised differences were sufficient and clearly below $10 \%$. Pump therapy was associated with a significantly lowered hazard ratio of between $26 \%$ and $44 \%$ for fatal/non-fatal coronary heart disease, fatal coronary heart disease, fatal cardiovascular disease, and all cause mortality.

\section{Analyses during study period}

Kaplan-Meier analysis disclosed significantly fewer $(\mathrm{P}=0.034)$ hypoglycaemic incidents with insulin pump therapy compared with multiple daily injections in patients with three or more incidents during seven years of follow-up (fig 2). There were no significant differences $(\mathrm{P}=0.9)$ in patients with one or two incidents during follow-up. The number of patients admitted to hospital for hypoglycaemic incidents during the study period was 148 in those treated with pump therapy and 
Table 2 | Hazard ratio (95\% confidence intervals) for various outcomes with insulin pump treatment compared with multiple daily injections (MDIs) in 18168 people with type 1 diabetes followed for mean of 6.8 years from 2005 to 2012

\begin{tabular}{|c|c|c|c|c|}
\hline & No with events (\%) & $\begin{array}{l}\text { Events/1000 } \\
\text { person years }\end{array}$ & $\begin{array}{l}\text { Hazard ratio* } \\
(95 \% \mathrm{Cl})\end{array}$ & $P$ value \\
\hline \multicolumn{5}{|c|}{ Major endpoints } \\
\hline \multicolumn{5}{|c|}{ Fatal/non-fatal coronary heart disease: } \\
\hline MDIs & $15727 / 1058(6.7)$ & 10.7 & 1.0 & \multirow{2}{*}{0.05} \\
\hline Pump & $2441 / 97(4.0)$ & 6.2 & 0.81 (0.66 to1.01) & \\
\hline \multicolumn{5}{|c|}{ Fatal/non-fatal cardiovascular disease: } \\
\hline MDIs & $15727 / 1294(8.2)$ & 13.1 & 1.0 & \multirow{2}{*}{0.2} \\
\hline Pump & 2441/129(5.3) & 8.3 & $0.88(0.73$ to1.06) & \\
\hline \multicolumn{5}{|c|}{ Fatal cardiovascular disease: } \\
\hline MDIs & $15727 / 517(3.3)$ & 5.1 & 1.0 & \multirow{2}{*}{0.005} \\
\hline Pump & $2441 / 29(1.2)$ & 1.8 & $0.58(0.40$ to 0.85$)$ & \\
\hline \multicolumn{5}{|c|}{ Total mortality: } \\
\hline MDIs & $15727 / 1109(7.1)$ & 11.0 & 1.0 & \multirow{2}{*}{0.007} \\
\hline Pump & $2441 / 83(3.4)$ & 5.3 & 0.73 (0.58 to 0.92$)$ & \\
\hline
\end{tabular}

\section{Secondary endpoints}

Fatal coronary heart disease:

\begin{tabular}{|c|c|c|c|c|}
\hline MDIs & $15727 / 453(2.9)$ & 4.5 & 1.0 & \multirow{2}{*}{0.004} \\
\hline Pump & $2441 / 24(1.0)$ & 1.5 & $0.55(0.36$ to 0.83$)$ & \\
\hline \multicolumn{5}{|c|}{ Fatal stroke: } \\
\hline MDIs & $15727 / 79(0.5)$ & 0.8 & 1.0 & \multirow{2}{*}{0.4} \\
\hline Pump & $2441 / 5(0.2)$ & 0.3 & $0.67(0.27$ to 1.67$)$ & \\
\hline \multicolumn{5}{|c|}{ Non-cardiovascular disease mortality: } \\
\hline MDIs & $15722 / 592(3.8)$ & 5.9 & 1.0 & \multirow{2}{*}{0.3} \\
\hline Pump & $2441 / 54(2.2)$ & 3.4 & 0.86 (0.64 to 1.13$)$ & \\
\hline
\end{tabular}

${ }^{*}$ Adjustment by stratification with fifths of propensity score including covariates of age, sex, diabetes duration, histories of cardiovascular disease, heart failure, atrial fibrillation, cancer, liver disease, mental disorders, education levels, and baseline values of $\mathrm{HbA}_{1}$, systolic and diastolic blood pressure, current smoking, physical activity, BMI, total and high density lipoprotein cholesterol, triglycerides, albuminuria, creatinine, renal insufficiency, antihypertensive drugs, lipid lowering drugs, aspirin, income, educational level, marital status and baseline year. used insulin pump therapy and do not know if the observed effect is attributable to continuous infusion of insulin or that some, if not all, of the effect is attributable to intensified glucose monitoring, increased motivation to control blood glucose, or a better knowledge about having diabetes type 1 .

\section{Comparison with other studies}

The reduced number of severe hypoglycaemic episodes could explain why insulin pump treatment is associated with a reduced cardiovascular mortality. Such episodes can trigger cardiac arrhythmias and coronary plaque rupture. ${ }^{29} 30$ Administration of insulin by continuous infusion has been reported to reduce the frequency of severe hypoglycaemia compared with administration of insulin by multiple daily injections. ${ }^{3132}$ There are indications that episodes of hypoglycaemia occur together with cardiac arrhythmia. ${ }^{33} 34$ Stahn and colleagues used continuous glucose measurements and continuous electrocardiograms for five days to monitor 30 people with type 2 diabetes and previous cardiovascular disease,. They found a 10 -fold increase in ventricular arrhythmias (mean $1.0 v 0.1$ ) during hypoglycaemic episodes. The corresponding figure for ventricular couplets was $7.6(41.7 v 5.5) .{ }^{35}$

Logically, having an insulin pump could result in a more stable blood glucose concentration than multiple daily injections. ${ }^{636}$ Often the history recorded by the pumps can be uploaded and displayed as a graph for purposes of trend analysis. ${ }^{37}$

\section{Mechanisms for results}

967 in those treated with injections, and the number of hypoglycaemic incidents was 206 and 1366, respectively (table $\mathrm{F}$ in appendix).

Analyses of updated mean $\mathrm{HbA}_{1 \mathrm{c}}$, as well as difference between baseline and final $\mathrm{HbA}_{1 \mathrm{c}}$, during the study period did not show significant differences between the treatments. Updated mean $\mathrm{HbA}_{1 \mathrm{c}}$ was 64 $\mathrm{mmol} / \mathrm{mol}(8 \%)$ in all those treated with pump therapy and $64 \mathrm{mmol} / \mathrm{mol}(8 \%)$ in all those treated with injections, and the mean decrease from baseline to final $\mathrm{HbA}_{1 \mathrm{c}}$ was $2.1 \mathrm{mmol} / \mathrm{mol}$ in both groups. Similarly, there was no difference in $\mathrm{HbA}_{1 \mathrm{c}}$ during the study period when patients were subdivided by combinations of fifths of baseline $\mathrm{HbA}_{1 \mathrm{c}}$, as well as baseline BMI, to exclude differences because of these baseline values (appendix table E).

\section{Discussion}

\section{Principal findings}

This is the first large study from a population based setting that documents the relation between insulin pump treatment and cardiovascular mortality. We studied individuals with type 1diabetes during a mean follow-up period of 6.8 years. Among 2441 of those treated with insulin pump therapy and 15727 treated with multiple daily injections, insulin pump treatment was associated with a reduction of $45 \%$ for fatal coronary heart disease, $42 \%$ for fatal cardiovascular disease, and 27\% for all cause mortality. We evaluated the patient who
There is a rationale for insulin pump treatment resulting in more stable blood glucose concentrations than multiple daily injections. Hypoglycaemia is a risk factor for cardiovascular events, particularly among high risk individuals. ${ }^{3839}$ We have recently found evidence that Swedish patients with type 1 diabetes and previous severe episodes of hypoglycaemia have an increased risk of mortality after a cardiovascular event. ${ }^{4}$ The Diabetes Control and Complication Trial of type 1 diabetes, however, found no significant association between frequent severe hypoglycaemic episodes and increased cardiovascular mortality among individuals in the intensive treatment group. ${ }^{40}$ The EURODIAB Prospective Complications Study of 2181 people with type 1 diabetes who were monitored for seven years also reported no association between baseline hypoglycaemia and the risk of cardiovascular disease. ${ }^{41}$ A retrospective analysis of a large cohort of people with type 1 diabetes a higher prevalence of cardiovascular disease in those with a history of repeated hypoglycaemic episodes. ${ }^{42}$

Another mechanism worth consideration is that treatment with insulin pump might lead to a lower frequency and duration of hyperglycaemia, corresponding to reduced long term occurrence of microvascular and and Complication Trial has shown that good glycaemic control for six years with follow-up for 11 years can significantly decrease the risk of any cardiovascular treated with insulin pump therapy, however, pointed to cardiovascular complications. The Diabetes Control 


\begin{tabular}{|c|c|c|c|c|}
\hline & \multicolumn{2}{|c|}{ Confounder with hazard ratio 1.3} & \multicolumn{2}{|c|}{ Confounder with hazard ratio 1.4} \\
\hline & \multicolumn{2}{|c|}{ Prevalence of confounder in pump group } & \multicolumn{2}{|c|}{ Prevalence of confounder in pump group } \\
\hline & $\overline{0.0}$ & 0.2 & $\overline{0.0}$ & 0.2 \\
\hline \multicolumn{5}{|c|}{ Fatal/non-fatal coronary heart disease } \\
\hline \multicolumn{5}{|c|}{ Prevalence of confounder in MDI group: } \\
\hline 0.0 & 0.81 (0.66 to 1.01$)$ & 0.76 (0.62 to 0.95$)$ & 0.81 (0.66 to 1.01$)$ & 0.75 (0.61 to 0.94$)$ \\
\hline 0.2 & 0.86 (0.70 to 1.07$)$ & 0.81 (0.66 to 1.01$)$ & 0.87 (0.71 to 1.09$)$ & $0.81(0.66$ to 1.01$)$ \\
\hline \multicolumn{5}{|c|}{ Fatal/non-fatal cardiovascular disease } \\
\hline \multicolumn{5}{|c|}{ Prevalence of confounder in MDI group: } \\
\hline 0.0 & 0.88 (0.73 to 1.06$)$ & 0.83 (0.69 to 1.00$)$ & 0.88 (0.73 to 1.06$)$ & $0.81(0.67$ to 0.98$)$ \\
\hline 0.2 & 0.93 (0.77 to 1.12$)$ & $0.88(0.73$ to 1.06$)$ & 0.95 (0.79 to 1.14$)$ & $0.88(0.73$ to 1.06$)$ \\
\hline \multicolumn{5}{|c|}{ Fatal cardiovascular disease } \\
\hline \multicolumn{5}{|c|}{ Prevalence of confounder in MDI group: } \\
\hline 0.0 & 0.58 (0.40 to 0.85$)$ & $0.55(0.38$ to 0.80$)$ & 0.58 (0.40 to 0.85$)$ & $0.54(0.37$ to 0.79$)$ \\
\hline 0.2 & $0.61(0.42$ to 0.90$)$ & 0.58 ( 0.40 to 0.85$)$ & 0.63 (0.43 to 0.92$)$ & $0.58(0.40$ to 0.85$)$ \\
\hline 0.4 & 0.65 (0.45 to 0.95$)$ & 0.61 (0.42 to 0.90$)$ & 0.67 (0.46 to 0.98$)$ & $0.63(0.43$ to 0.92$)$ \\
\hline 0.6 & 0.68 (0.47 to 1.00$)$ & 0.65 (0.45 to 0.95$)$ & 0.72 (0.50 to 1.05$)$ & $0.67(0.46$ to 0.98$)$ \\
\hline 0.8 & 0.72 (0.50 to 1.05$)$ & 0.68 (0.47 to 1.00$)$ & 0.77 (0.53 to 1.12$)$ & $0.72(0.50$ to 1.05$)$ \\
\hline \multicolumn{5}{|c|}{ Total mortality } \\
\hline \multicolumn{5}{|c|}{ Prevalence of confounder in MDI group: } \\
\hline 0.0 & 0.73 (0.58 to 0.92$)$ & 0.69 (0.55 to 0.87$)$ & 0.73 (0.58 to 0.92$)$ & 0.68 (0.54 to 0.85$)$ \\
\hline 0.2 & 0.77 (0.61 to 0.97$)$ & 0.73 (0.58 to 0.92 ) & 0.79 (0.63 to 0.99$)$ & 0.73 (0.58 to 0.92$)$ \\
\hline 0.4 & 0.82 (0.65 to 1.03$)$ & 0.77 (0.61 to 0.97$)$ & 0.85 (0.67 to 1.07 ) & 0.79 (0.63 to 0.99$)$ \\
\hline 0.6 & 0.86 (0.68 to 1.08$)$ & 0.82 (0.65 to 1.03$)$ & $0.90(0.72$ to 1.14$)$ & $0.85(0.67$ to 1.07$)$ \\
\hline
\end{tabular}

disease event by $42 \%$ and the risk of non-fatal myocardial infarction, stroke, or death from cardiovascular disease by $57 \% .^{2}$ At the group level, it is clear that sensor-augmented pump therapy provides better metabolic control than multiple daily injections in adults with type 1 diabetes. A study in the United States and Canada randomised 329 adults with type 1 diabetes to insulin with sensor-augmented pump therapy or multiple daily injections. After one year, $\mathrm{HbA}_{1 \mathrm{c}}$ had been reduced by $1.0 \%$ from baseline among those who had been randomised to sensor-augmented pump therapy. The corresponding figure for injections was $0.4 \%$, with a significant difference..$^{43} \mathrm{~A}$ review of the literature in 2010 found some evidence that insulin pump therapy without continuous glucose monitoring could be better than multiple daily injections for glycaemic control in people with type 1 diabetes, with adjustment for baseline $\mathrm{HbA}_{1 \mathrm{c}}{ }^{7}$ This study gave no information on sensor use, but it was uncommon in Sweden during the period.

\section{Strengths of the study}

This study included a large number of participants. Each individual with type 1 diabetes who was entered as being treated with insulin pump therapy or multiple daily injections was reported to the Swedish National Diabetes Register by local units. Nobody was excluded from the study during follow-up, and we have valid information for almost every Swede who has been diagnosed with type 1 diabetes, ${ }^{12}$ as well as information regarding the occurrence of cardiovascular disease and death outcomes using established national registers. ${ }^{17} 18$ The propensity score allowed for balancing 36 covariates between the insulin pump and multiple daily injection groups, including strong cardiovascular risk factors and important social data-there were only non-significant differences and small standardised differences for all covariates. The stratification into fifths of the score for adjustment at the Cox regressions permitted use of all available patients in the study. Score stratification with fifths is sometimes regarded as causing less residual confounding than not using fifths. ${ }^{20}{ }^{21}$ Subgroup analyses allowed for further verification of the study results in patients with no previous cardiovascular disease or other serious concomitant disease; the marker was low BMI. The analysis of a somewhat smaller sample with complete data and no imputation confirmed our results, which indicated that missing data were random.

We observed clinical practice in Sweden at the time of the study. There were no strict guidelines for switching from multiple daily injections to insulin pump therapy. Among possible reasons for a physician to recommend that an individual with type 1 diabetes switch treatment are unsatisfactory glycaemic control with high $\mathrm{HbA}_{1 \mathrm{c}}$, large variations in blood glucose concentrations, or the need to improve quality of life by administering insulin more flexibly. In Sweden treatment by pump and multiple daily injections is covered by healthcare providers, there are no additional costs for the patient.

Our analysis of the effect of a hypothetical unmeasured confounder showed that this effector would have to be large (hazard ratios of 1.3-1.4) and with a prevalence of $80 \%$ or more in the injection group but no presence in the insulin pump group to eliminate the 

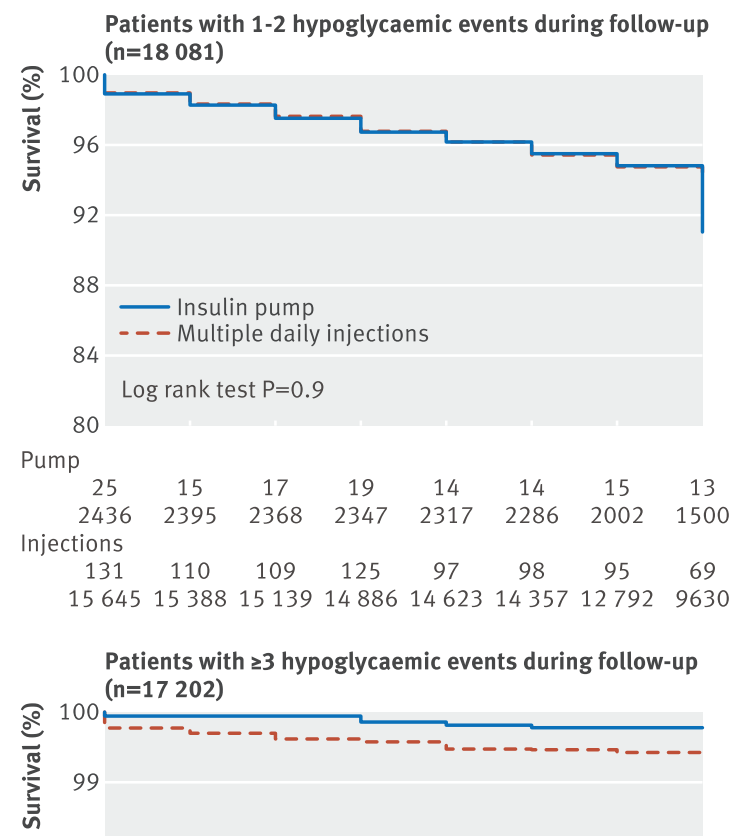

98

$$
97
$$

$$
96
$$$$
\text { Log rank test } \mathrm{P}=0.034
$$$$
95
$$$$
0
$$$$
1
$$$$
2
$$$$
3
$$$$
\text { Pump }
$$$$
1
$$$$
0
$$$$
\begin{gathered}
0 \\
280 \quad 2
\end{gathered}
$$$$
34
$$$$
5
$$

\section{6}

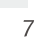

Injections

$\begin{array}{llllllll}32 & 11 & 13 & 6 & 13 & 2 & 4 & 1\end{array}$

Fig 2 | Kaplan-Meier survival curves for first incident hypoglycaemic events in patients with type 1 diabetes during seven years of follow-up. No of cases and individuals at risk are given for each group

significant findings for risk of fatal coronary heart disease and cardiovascular disease (table 3). ${ }^{25-28}$ In light of these simulations, and that we are not aware of any strong unmeasured risk factor for coronary heart disease that is likely to be severely unbalanced between those using pump and injections, we believe we have documented a true effect. Obviously we need more data before we can state without reasonable doubt that pump use results in a lower risk of coronary heart disease or cardiovascular disease.

\section{Limitations of the study}

One limitation of the study was that we had no information on duration of insulin pump treatment before study baseline, although our aim was to analyse outcomes from baseline during a long term follow-up period. If the mechanism for the preventive effect of insulin pump treatment on cardiovascular mortality is through a reduced frequency of lethal arrhythmia, we would expect adjustment for the duration of insulin pump treatment to have little or no effect on our hazard ratios. If instead the mechanism is through events with an induction latency time of a year or more, such as plaque formation, adjustment for duration of insulin pump treatment would give even stronger associations than we found. We adjusted the hazard ratio between insulin pump treatment and cardiovascular mortality for baseline values of $\mathrm{HbA}_{1 \mathrm{c}}$. As some patients had used insulin pumps for some time at baseline, this means that the adjustment might eliminate some of the effect. That is, if this source of error did not exist, we would have estimated the protective effect of insulin pump treatment on cardiovascular mortality to be larger than we now found. We did not adjust for $\mathrm{HbA}_{1 \mathrm{c}}$ after baseline as that would be adjusting for a possible mediating factor. Separate analyses of updated mean $\mathrm{HbA}_{1 \mathrm{c}}$ during the study, or the change between baseline and final $\mathrm{HbA}_{1 \mathrm{c}}$, however, showed no significant differences between the treatment groups.

Mediating factors, to one extent or another, for the effect of insulin pump treatment on cardiovascular mortality might be increased frequency of glucose monitoring, as well as more appropriate actions at various blood glucose concentrations. Changing from multiple daily injections to insulin pump treatment is accompanied by education about insulin pump treatment, which could be useful in reducing the number of episodes of hypoglycaemia and hyperglycaemia. Moreover, having a pump with the opportunities it offers to fine tune the administration of insulin might itself be an instructive factor. Thus, some of the effect of pump therapy on risk of cardiovascular disease could have been achieved by intensified training of the individual about the disease $\mathrm{e}^{44}$ to improve blood glucose monitoring and achieve a better balance between insulin administration, food intake, and physical activity.

\section{Conclusions}

This nationwide observational study of individuals with type 1 diabetes shows that treatment with an insulin pump was associated with a considerable reduction in risk of fatal coronary heart disease, fatal cardiovascular disease, and all cause mortality. Whether the results reflect the physiological consequences of insulin pump treatment, the clinical management that pump users receive, or the educational aspects of having the pump remains elusive.

We thank all regional Swedish National Diabetes Register

coordinators, contributing nurses, physicians, and patients who contributed to the success of this study. The Swedish Society of Diabetology and the Swedish Diabetes Association, a patient advocacy group, support the Swedish National Diabetes Register. The results and views expressed in the study represent those of the authors and not necessarily those of the Swedish Medical Products Agency, at which one of the authors (BZ) is employed. The study was presented at the 50th European Association for the Study of Diabetes (EASD) Annual Meeting, Vienna, Austria, September 2014.

Contributors: IS, JC, and SG researched the data; IS and JC performed the statistical analyses; IS, JC, and SG wrote the article, contributed to the discussion, and reviewed and edited the article. BE, AR, KE-O, A-MS, BZ, TA, ML-O, and JJ contributed to the discussion and reviewed and edited the article. SG is guarantor.

Funding: This study received funding from the European Association for the study of diabetes. The Swedish National Diabetes Register is funded by the Swedish Association of Local Authorities and Regions. Competing interests: All authors have completed the ICMJE uniform disclosure form at www.icmje.org/coi_disclosure.pdf and declare: KE-O has received fees from Sanofi and Novo Nordisk for lectures 
outside the submitted work; ML-O lectures about diabetology and has been paid by different pharmaceutical companies.

Ethical approval: The study was approved by the regional ethical review board at the University of Gothenburg. All individuals with diabetes give their informed consent before being entered.

Data sharing: No additional data available, but data from this study are available on request.

Transparency: The lead author affirms that the manuscript is an honest, accurate, and transparent account of the study being reported; that no important aspects of the study have been omitted; and that any discrepancies from the study as planned have been explained.

This is an Open Access article distributed in accordance with the Creative Commons Attribution Non Commercial (CC BY-NC 4.0) license, which permits others to distribute, remix, adapt, build upon this work non-commercially, and license their derivative works on different terms, provided the original work is properly cited and the use is non-commercial. See: http://creativecommons.org/licenses/ by-nc/4.0/

1 Lind M, Svensson AM, Kosiborod M, et al. Glycemic control and excess mortality in type 1 diabetes. N Engl I Med 2014:371:1972-82.

2 Nathan DM, Cleary PA, Backlund JY, et al. Intensive diabetes treatment and cardiovascular disease in patients with type 1 diabetes. N Engl Med 2005;353:2643-53.

3 Eeg-Olofsson K, Cederholm J, Nilsson PM, et al. Glycemic control and cardiovascular disease in 7454 patients with type 1 diabetes: an observational study from the Swedish National Diabetes Register (NDR). Diabetes Care 2010:33:1640-6.

4 Lung TW, Petrie D, Herman WH, et al. Severe hypoglycemia and mortality after cardiovascular events for Type 1 diabetic patients in Sweden. Diabetes Care 2014:37:2974-81.

5 Plotnick LP, Clark LM, Brancati FL, et al. Safety and effectiveness of insulin pump therapy in children and adolescents with type 1 diabetes. Diabetes Care 2003;26:1142-6.

6 Bruttomesso D, Pianta A, Crazzolara D, et al. Continuous subcutaneous insulin infusion (CSII) in the Veneto region: efficacy, acceptability and quality of life. Diabet Med 2002;19:628-34

7 Misso ML, Egberts KJ, Page M, et al. Continuous subcutaneous insuli infusion (CSII) versus multiple insulin injections for type 1 diabetes mellitus. Cochrane Database Syst Rev 2010;1:CD005103.

8 Fatourechi MM, Kudva YC, Murad MH, et al. Hypoglycemia with intensive insulin therapy: a systematic review and meta analyses of randomized trials of CSII versus MDI. J Clin Endocrinol Metab 2009;94:729-40.

9 Jeitler K, Horvath K, Berghold A, et al Continuous subcutaneous insulin infusion versus multiple daily insulin injections in patients with diabetes mellitus: systematic review and meta-analysis. Diabetologia 2008;51:941-51.

10 Monami M, Lamanna C, Marchionni N, et al. CSII versus MDI in type 1 diabetes: a meta-analysis. Acta Diabetol 2010;47:77-81

11 Fredheim S, Johansen A, Thorsen SU, et al. Nationwide reduction in the frequency of severe hypoglycemia by half. Acta Diabetol 2014;52:591-9.

12 Annual Report 2013. Swedish National Diabetes Register (NDR), Sweden, 2013. www.ndr.nu.

13 Gudbjörnsdottir S, Cederholm J, Nilsson PM, et al, for the Steering Committee of the National Diabetes Register. The National Diabetes Register in Sweden. An implementation of the St. Vincent Declaration for Quality Improvement in Diabetes Care. Diabetes Care 2003;26:1270-6.

14 Cederholm J, Eeg-Olofsson K, Eliasson B, et al; Swedish National Diabetes Register. A new model for 5 -year risk of cardiovascular disease in type 1 diabetes; from the Swedish National Diabetes Register (NDR). Diabet Med 2011;28:1213-20

15 Eliasson B, Gudbjörnsdottir S. Diabetes care-improvement through measurement. Diabetes Res Clin Pract 2014;106(suppl 2):S291-4.

16 Geistanger A, Arends S, Berding C, et al. Statistical methods for monitoring the relationship between the IFCC reference measurement procedure for hemoglobin $\mathrm{A}_{1 c}$ and the designated comparison methods in the United States, Japan, and Sweden. Clin Chem 2008; 54:1379-85.

17 Merlo J, Lindblad U, Pessah-Rasmussen $\mathrm{H}$, et al. Comparison of different procedures to identify probable cases of myocardial infarction and stroke in two Swedish prospective cohort studies using local and national routine registers. Eur J Epidemiol 2000;16:235-43.

18 Tunstall-Pedoe H, Kuulasmaa K, Amouyel P, et al. Myocardial infarction and coronary deaths in the World Health Organization MONICA Project. Registration procedures, event rates, and case-fatality rates in 38 populations from 21 countries in four continents. Circulation 1994:90:583-612

19 Berglund P, Heeringa S, eds. Multiple imputation of missing data using SAS. SAS Institute, July 2014
20 D’Agostino RB Jr. Propensity score methods for bias reduction in the comparison of a treatment to a non-randomized control group. Stat Med 1998:17:2265-81.

21 Faries DE, Leon AC, Haro JM, eds. Analysis of observational health care data using SAS. SAS Institute, February 2010.

22 Austin PC, Grootendorst P, Anderson GM. A comparison of the ability of different propensity score models to balance measured variables between treated and untreated subjects: a Monte Carlo study. Stat Med 2007;26:734-53.

23 Estimating Cox regression models with Proc Phreg. In: Allison PD, ed Survival analysis using SAS. A practical guide. Chapter 5. 2nd ed. SAS Institute, April 2010.

24 Lin DY, Psaty BM, Kronmal RA. Assessing the sensitivity of regression results to unmeasured confounders in observational studies. Biometrics 1998;54:948-63

25 Abdollah F, Schmitges J, Sun M, et al. Comparison of mortality outcomes after radical prostatectomy versus radiotherapy in patients with localized prostate cancer: a population-based analysis. Int J Urol 2012;19:836-44

26 Eklind-Cervenka M, Benson L, Dahlström U, et al. Association of candesartan vs losartan with all-cause mortality in patients with heart failure. JAMA 2011;305:175-82

27 Sooriakumaran P, Nyberg T, Akre O, et al. Comparative effectiveness of radical prostatectomy and radiotherapy in prostate cancer: observational study of mortality outcomes. BMJ 2014;348:g1502

28 Ekström N, Cederholm J, Zethelius B, et al. Aspirin treatment and risk of first incident cardiovascular diseases in patients with type 2 diabetes: an observational study from the Swedish National Diabetes Register. BMJ Open 2013;3:e002688.

29 Pickup JC, Sutton AJ. Severe hypoglycaemia and glycaemic control in type 1 diabetes: meta-analysis of multiple daily insulin injections compared with continuous subcutaneous insulin infusion. Diabet Med 2008;25:765-74

30 Arbab-Zadeh A, Nakano M, Virmani R, et al. Acute coronary events. Circulation 2012;125:1147-56

31 Bode BW, Steed RD, Davidson PC. Reduction in severe hypoglycemia with long-term continuous subcutaneous insulin infusion in type 1 diabetes. Diabetes Care 1996:19:324-7.

32 Yeh HC, Brown TT, Maruthur N, et al. Comparative effectiveness and safety of methods of insulin delivery and glucose monitoring for diabetes mellitus: a systematic review and meta-analysis. Ann Intern Med 2012;157:336-47.

33 Chow E, Bernjak A, Williams S, et al. Risk of cardiac arrhythmias during hypoglycemia in patients with type diabetes and cardiovascular risk. Diabetes 2014;63:1738-47.

34 Ray KK, Seshasai SR, Wijesuriya S, et al. Effects of intensive control of glucose on cardiovascular outcomes and death in patients with diabetes mellitus Lancet 2009:373:1765-72.

35 Stahn A, Pistrosch F, Ganz X, et al. Relationship between hypoglycemic episodes and ventricular arrhythmias in patients with type 2 diabetes and cardiovascular diseases: silent hypoglycemias and silent arrhythmias. Diabetes Care 2014;37:516-20.

36 Health Quality Ontario. Continuous subcutaneous insulin infusion (CSII) pumps for type 1 and type 2 adult diabetic populations: an evidence-based analysis. Ont Health Technol Assess Ser 2009;9:1-58.

37 Pickup JC. Insulin-pump therapy for type 1 diabetes mellitus. N Engl J Med 2012;366:1616-24

38 Duckworth W, Abraira C, Moritz T, et al. Glucose control and vascular complications in veterans with type 2 diabetes. N Engl J Med 2009;360:129-39

39 Mellbin LG, Rydén L, Riddle MC, et al. Does hypoglycaemia increase the risk of cardiovascular events? A report from the ORIGIN trial. Eur Heart/ 2013;34:3137-44.

40 Diabetes Control and Complications Trial Research Group Hypoglycemia in the diabetes control and complications trial. Diabetes 1997;46:271-86

41 Gruden G, Barutta F, Chaturvedi N, et al. Severe hypoglycemia and cardiovascular disease incidence in type 1 diabetes: the EURODIAB Prospective Complications Study. Diabetes Care 2012;35:1598-604.

42 Gimenez M, Lopez JJ, Castell C, et al. Hypoglycaemia and cardiovascular disease in type 1 diabetes. Results from the Catalan National Public Health registry on insulin pump therapy. Diabetes Res Clin Pract 2012;96:23-5.

43 Bergenstal RM, Tamborlane WV, Ahmann A, et al. Effectiveness of sensor augmented insulin pump therapy in type 1 diabetes. $N$ EnglJ Med 2010;363:311-20.

44 Snow R, Humphrey C, Sandall J. What happens when patients know more than their doctors? Experiences of health interactions after diabetes patient education: a qualitative patient-led study. BMJ Open 2013;3:e003583.

(c) BMJ Publishing Group Ltd 2015

Appendix: Supplementary tables A-F [posted as supplied by author] 